\title{
The American Phytopathological Society Elected and Appointed Officers, Representatives, and Committees for 2008
}

Council

President: R. D. Martyn

President-Elect: J. W. Moyer

Vice President: B. J. Christ

Immediate Past President: J. E. Leach

Secretary: D. T. Beadle

Treasurer: R. C. Rowe

Senior Councilor-at-Large: W. F. Wilcox

Intermediate Councilor-at-Large: G. W. Moorman

Junior Councilor-at-Large: M. J. Boehm

Editor-in-Chief, APS PRESS: M. L. Daughtrey

Editor-in-Chief, Molecular Plant-Microbe Interactions: J. D. Walton

Editor-in-Chief, Phytopathology: R. L. Gilbertson

Editor-in-Chief, Phytopathology News: J. E. Loper

Editor-in-Chief, Plant Disease: A. P. Keinath

Councilor of the Caribbean Division: L. A. Calvert

Councilor of the North Central Division: R. Hammerschmidt

Councilor of the Northeastern Division: R. L. Wick

Councilor of the Pacific Division: M. Putnam

Councilor of the Potomac Division: K. L. Everts

Councilor of the Southern Division: J. C. Rupe

Ex officio: APS Foundation Rep: A. R. Chase

Executive Vice President: S. C. Nelson

\section{Representatives}

American Association for the Advancement of Science: D. G. Gilchrist

American Institute of Biological Sciences: B. J. Christ

American Type Culture Collection: C. T. Bull

Coalition on Funding Agricultural Research: J. L. Sherwood

Council for Agricultural Science \& Technology: T. B. Sutton

Intl. Society for Aboriculture: G. W. Hudler

International Society for Plant Pathology: Representative: T. A. Evans.

Members: G. S. Abawi, J. H. Andrews, R. A. Bennett, L.A. Calvert, K. F.

Cardwell, J. C. Correll, J. W. Moyer, R. C. Ploetz, R. S. Zeigler

International Union of Microbiological Societies: C. T. Bull

Mycological Society of America: C. M. Stiles

National Council for Science and the Environment: K. L. Pernezny

Society of Environmental Toxicology and Chemistry: M. Simini

Society of Nematologists: T. L. Niblack

APS Historian: P. D. Peterson

APS Foundation, Board of Directors

Chair: A. R. Chase. Vice Chair: M. L. Gleason. Treasurer: R. C. Rowe. G. S. Abawi, A. M. Alvarez, J. Amador, C. J. D'Arcy, W. E. Fry, G. L. Schumann. Ex-Officio (non-voting): N. S. Dufault

Office of Electronic Communications (OEC) Board

Director: D. M. Eastburn. APSnet Feature Editor: M. L. Elliott. D. A. Glawe, G. G. Grove, S. Kang, K. J. Martin, P. M. Sforza, K. C. Steddom. Ex-Officio Voting: A. B. Baudoin

Office of International Programs (OIP) Advisory Board

Director: S. A. Miller. M. Babadoost, S. Kamoun, J. H. McBeath, A. C. Schilder. Ex-Officio (non-voting): W. E. Fry

Office of Industry Relations (OIR) Board

Director: B. D. Olson. C. M. Becker, L. Fought, P. T. Himmel, C. L. Kohls, C. T. Schiller, V. Spadafora, W. L. Wiebe, M. D. Wiglesworth, H. L. Ypema. Ex-Officio Voting: M. R. Schwarz, E. C. Tedford

Office of Public Relations and Outreach (OPRO) Board

Director: D. J. Jardine. G. S. Abawi, R. H. Brlansky, J. P. Damicone, M. N. Donofrio, M. A. Draper, M. A. Langham, C. T. Schiller, K. T. Schneider. Ex-Officio, APSnet Feature Editor: M. L. Elliott. Ex-Officio Voting: J. Fletcher, J. E. Leach

Public Policy Board (PPB)

Chair: J. Fletcher. N. B. Carroll, B. J. Christ, W. E. Dolezal, S. E. Gold, S. H. Hulbert, J. D. MacDonald, R. D. Martyn, J. W. Moyer, J. R. Steadman. Ex-Officio (non-voting): D. J. Jardine
Publications Board

Chair: M. E. Daub. A. B. Baudoin, M. L. Daughtrey, D. M. Eastburn, D. S. Egel, R. L. Gilbertson, A. P. Keinath, J. E. Loper, M. E. Matheron, J. D. Walton

Scientific Programs Board (SPB)

Director: S. T. Adkins. Program Chair: J. W. Moyer. Vice Chair: B. J. Christ. Section Chairs: M. J. Boehm, A. O. Charkowski, L. M. Ciuffetti, C. Cowger, A. K. Culbreath, D. A. Shah. Workshop Chair: J. W. Buck. A. B. Baudoin, W. F. Wilcox

Division Officers

Caribbean Division: President: Y. Martinez Zubiaur. Vice President: L. E. Datnoff. Past President: O. A. Moreno-Valenzuela. Secretary-Treasurer: R. H. Brlansky

North Central Division: President: C. Hollingsworth. Secretary-Treasurer: L. J. Giesler

Northeastern Division: President: C. A. Smith. Vice President: D. R. Cooley. Immediate Past President: W. H. Elmer. Secretary-Treasurer: J. A. LaMondia

Pacific Division: President: W. D. Gubler. Past President: L. D. Porter. Secretary-Treasurer: C.-L. Xiao

Potomac Division: President: D. P. Roberts. Vice President: I. A. Zasada. Immediate Past President: J. L. Norelli. Secretary-Treasurer: D. G. Schmale

Southern Division: President: C. A. Clark. President-Elect: K. W. Seebold. Vice President: R. C. Kemerait. Past President: C. S. Rothrock. SecretaryTreasurer: T. S. Isakeit

\section{APS PRESS Editorial Board}

Editor-in-Chief: M. L. Daughtrey. Associate Editor-in-Chief: T. C. Paulitz. Aquisitions Editors: B. J. Jacobsen, L. V. Madden. Senior Editors: J. K. Brown, J. H. Graham, S. T. Koike, B. B. McSpadden Gardener, S. A. Miller, G. P. Munkvold, M. E. Palm-Hernandez, A. C. Schilder, H. F. Schwartz. Ex-Officio (non-voting): R. C. Rowe

Illustrations of Plant Pathogens and Diseases: Chair: A. C. Schilder

Standardization of Common Names of Plant Diseases: Chair: B. A. Fortnum

\section{Editors of Molecular Plant-Microbe Interactions}

Editor-in-Chief: J. D. Walton. Senior Editors: J. R. Alfano, J. P. Carr, G. De Lorenzo, P. Franken, S. Genin, G. Gheysen, M. Gijzen, S. E. Lindow, R. Loria, J.-P. Metraux, C. W. Ronson, C. L. Schardl, P. D. Spanu, K. Szczyglowski, B. P. H. J. Thomma, L. Walling. Indexing Editor: T. Kommedahl. Associate Editors: K. Akimitsu, M. Barnett, P. Birch, O. Borras-Hidalgo, A. O. Charkowski, K.-R. Chung, U. Conrath, A. Devoto, A. C. Di Pietro, B. Ding, R. Geurts, F. Krajinski, H. Kuester, K. N. Lambert, M. Lorito, Y. Moënne-Loccoz, G. E. D. Oldroyd, G. M. Preston, N. Requena, S. Sato, P. Schweizer, J. Shah, G. Smant, P. Solomon, X. Tang, J. Ton, J. Valkonen, G. Van Den Ackerveken, J. Verchot-Lubicz, P. Veronese

\section{Editors of Phytopathology}

Editor-in-Chief: R. L. Gilbertson. Senior Editors: V. C. Blok, N. J. Grunwald, J. B. Jones, D. Y. Kobayashi, F. J. Louws, N. McRoberts, T. J. Michailides, E. B. Nelson, N. D. Paul, A. L. N. Rao, D. A. Samac, S. P. Singh, G. Sunter. Associate Editors: T. B. Adhikari, D. J. Bailey, P. J. Balint-Kurti, J. D. Barak, C. Cowger, R. G. Dietzgen, M. L. Farman, R. C. Hamelin, A. V. Karasev, G. R. Knudsen, K. H. Lamour, C. Lannou, D. V. Mavrodi, J. D. Palumbo, J. Polashock, A. Rezaian, P. Timper, G. E. Vallad, L. Willocquet

\section{Editors of Plant Disease}

Editor-in-Chief: A. P. Keinath. Senior Editors: R. E. Baird, X. Chen, R. M. Davis, M. E. Hilf, P. S. McManus, E. A. Milus, P. V. Oudemans, M. G. Redinbaugh, B. B. Shew, R. N. Trigiano, R. R. Walcott, W. M. Wintermantel, G. Y. Yuen. Focus Editor: T. Kommedahl. Feature Editor: T. L. Niblack. Disease Notes Assigning Editor: S. T. Adkins. Associate Editors: J. A. Abad, K. Baumgartner, W. W. Bockus, C. A. Bradley, J. K. Brown, J. W. Buck, D. J. Chitwood, D. R. Cooley, L. del Rio, A. E. Dorrance, P. D. Esker, T. G. Fetch, Jr., F. E. Gildow, R. M. Harveson, M. Lapidot, R. E. Lee, D. S. Mueller, N. Peres, S. J. Pethybridge, W. F. Pfender, B. M. Pryor, G. Schnabel, D. A. Shah, N. Shishkopf, N. R. Walker, F. P. Wong

Editors of Plant Health Instructor/APSnet Education Center

Editor-in-Chief: A. B. Baudoin. Senior Editors: M. C. Becktell, A. Brooks Gould, M. A. Langham, S. J. Pethybridge, K. L. Stevenson, E. C. Tedford, M. T. Windham, J. Xu. Associate Editors: J. A. Abad, N. Peres 
Editors of Plant Health Progress

Editor-in-Chief: M. E. Matheron. Senior Editors: M. C. Black, G. T. Browne, D. A. Cuppels, J. J. Farrar, T. Kuhar, M. W. Olsen, R. K. D. Peterson, P. M. Phipps, N. A. Tisserat. Associate Editors: C. Cowger, D. M. Ferrin, D. O. Gilrein, K. L. Ivors, C. Nischwitz, C. M. Ocamb, J. E. Polston, P. B. Schultz, K. C. Steddom, I. A. Zasada. Ex-Officio, APSnet Feature Editor: M. L. Elliott. APS PRESS Editor-in-Chief: M. L. Daughtrey

\section{Editors of Plant Disease Management Reports}

Editor-in-Chief: D. S. Egel. Assigning Editors: C. M. Becker, D. R. Cooley, R. F. Davis, A. Henn, C. R. Hollingsworth, C. S. Johnson, R. C. Kemerait, N. Lalancette, R. P. Larkin, C. Y. Warfield, F. P. Wong. Section Editors: C. A. Bradley, M. A. Draper, L. J. du Toit, P. F. Harmon, M. M. Kennelly, M. F. R. Khan, C. S. Kousik, D. B. Langston, A. Mengistu, J. C. Mertely, T. M. Momol, B. D. Olson, G. B. Padgett, S. L. Rideout, P. D. Roberts, K. W. Seebold, C. A. Wyenandt

\section{Phytopathology News Advisory}

Editor-in-Chief: J. E. Loper. D. G. Fernando, M. Lorito, G. W. Moorman, D. G. Schmale

\section{AdHoc Committees}

Auxiliary Meetings and Workshops: Chair: G. C. Bergstrom. S. T. Adkins, J. W. Buck, R. C. Rowe, G. C. Wisler

Centennial Coordinating: Chair: J. W. Moyer. B. J. Christ, C. J. D’Arcy, J. E. Leach, R. D. Martyn, R. C. Rowe, A. H. Tally

Future of Education in Plant Pathology: Chair: J. D. MacDonald. C. Allen, W. R. Jacobi, S. Kelemu, J. W. Moyer, T. D. Murray, K. Ong, C. A. Pearson, J. L. Sherwood, A. K. Vidaver

International Programs: Chair: R. C. Ploetz. T. A. Evans, N. Grunwald, J. McBeath, S. A. Miller, J. W. Moyer, G. Ponciano

ISF Collaboration on Codification of Plant Races/Strains Commonly Used in the Seed Industry: Chair: P. T. Himmel. W. E. Dolezal, L. J. DuToit, V. Grimault, C. J. Kurowski, R. Ranganathan, C. M. Sandlin, K. van Ettekoven, K. M. Webb

Leadership and Volunteer Development: Chair: A. H. Tally. P. F. Harmon, T. K. Mitchell, J. B. Ristaino, N. L. Russo, J. L. Starr

Profession of Plant Pathology; Status and Future Prospects: Chair: D. M. Gadoury. J. H. Andrews, K. Baumgartner, T. J. Burr, M. M. Kennelly, A. Lichens-Park, J. L. Riggs, S. S. Savary, H. Scherm, G.-L. Wang

Working Group on Culture Collections: Chair: J. B. Jones. R. A. Bennett, C. M. Catranis, J. Chen, F. M. Dugan, J. H. Hill, D. Y. Kobayashi, N. W. Schaad, N. A. Tisserat

\section{Special Committees}

Advisory Committee on Threatening Plant Diseases: Chair: J. L. Sherwood. W. E. Dolezal, M. A. Draper, J. Fletcher, L. J. Giesler, S. H. Kim, J. E. Leach, C. A. Levesque, D. G. Luster, L. L. McDaniel, M. H. Royer, K. L. Smith, J. P. Stack

Centennial Planning: Chair: C. J. D'Arcy. R. R. Belanger, M. N. Cline, R. C. Gergerich, P. D. Peterson, E. N. Rosskopf, K. L. Schroeder, L. Sequeira, E. L. Stromberg, A. H. Tally

Emerging Diseases and Pathogens: Chair: D. G. Luster. A. M. Alvarez, R. A. Bennett, P. H. Berger, M. R. Bonde, J. K. Brown, R. Bulluck, W. Chun, V. D. Damsteegt, J. Fletcher, N. Grunwald, J. Hammond, J. S. Hartung, D. M. Huber, C. A. Ishimaru, G. Kuldau, L. Levy, R. F. Line, L. V. Madden, C. W. Magill, R. R. Martin, D. C. McGee, F. W. Nutter, N. W. Schaad, D. R. Smith, J. P. Stack, S. A. Tolin, A. T. Tschanz, A. K. Vidaver, G. C. Wisler, C. P. Woloshuk, X.-B. Yang

Meeting of the Americas: Chair: L. A. Calvert. J. Amador, R. H. Brlansky, E. R. French, Z. K. Punja

\section{Society Internal Relations Committees}

Awards and Honors: Chair: R. C. Seem. Immediate Past Chair: C. E. Windels. Vice Chair: C. L. Schardl. B. W. Falk, M. P. McMullen, L. W. Timmer, D. M. Weller

Committee on Committees: Chair: W. F. Wilcox. G. W. Moorman, M. J. Boehm

Executive: Chair: R. D. Martyn. D. T. Beadle, B. J. Christ, J. E. Leach, J. W. Moyer, R. C. Rowe, W. F. Wilcox

Financial Advisory: Chair: R.C. Rowe. D. T. Beadle, M. J. Boehm, B. J. Christ, M. L. Daughtrey, R. D. Martyn, J. W. Moyer, J. C. Rupe, W. F. Wilcox

\section{Society General Policies Committees}

Collections and Germplasm: Chair: W. Chen. Immediate Past Chair: J. Chen. Vice Chair: A. Wiest. L. E. Cadle-Davidson, M. D. Coffey, F. M. Dugan, B. J. Goates, E. W. Jackson, J. B. Jones, S. Li, D. L. Long

Early Career Professionals: Chair: D. G. Schmale. Immediate Past Chair: B. K. Scholz-Schroeder. Vice Chair: Z. Kanaan-Atallah. M. E. Ambroson, R. Bulluck, N. J. Catlin, T. E. Coram, S. L. Giammaria, P. F. Harmon, C. E. Jahn, L. M. Keith, W. Liu, P. R. Northover, K. Ong, M. E. Ordonez,
L. I. Santamaria, K. T. Schneider, V. Toussaint, M. R. Vazquez, K. M. Webb, A. Westphal, C. M. Wistrom, L. Xing

Extension: Chair: M. M. Kennelly. Immediate Past Chair: D. K. Malvick. Vice Chair: K. Ong. C. J. Balbalian, G. S. Bender, C. A. Bradley, M. E. L. Burrows, E. D. De Wolf, A. DeMarsay, J. S. Engle, P. D. Esker, D. M. Ferrin, L. J. Giesler, M. A. Grabowski, B. K. Gugino, P. F. Harmon, A. Henn, T. A. Jackson, C. Lapaire Harmon, C. J. Mickler, S. C. Nelson, L. E. Osborne, J. M. Rees, A. E. Robertson, B. E. Ruden, G. E. Ruhl, A. C. Schilder, K. C. Steddom, A. U. Tenuta, S. J. Vasquez, J. WilliamsWoodward, J. E. Woodward, A. D. Ziems

Graduate Student: Chair: N. S. Dufault. Immediate Past Chair: B. A. Edmunds. Vice Chair: C. A. Gallup. F. E. Bartz, G. C. Bernard, K. Burr, J. L. Calcote, A. Chambers, J. Cobb, S. J. Colucci, M. L. Cromwell, J. D. Dominiak, S. L. Giammaria, C. E. Jahn, S. C. Marine, H. A. Olson, A. R. Records, C. L. Rivard, D. L. Smith, K. E. Tilley, M. E. Vega Sanchez, C. M. Wallis, O. S. D. Wally, K. R. Whitten Buxton

Industry: Chair: L. Fought. Immediate Past Chair: B. D. Olson. Vice Chair: R. S. Bounds. K. S. Arthur, D. T. Beadle, J. N. Bruhn, P. D. Brune, W. E. Dolezal, A. P. Hert, V. Jurin, M. C. Klapproth, P. J. Kuhn, H. Mellinger, H. Morton, C. L. Palmer, L. A. Payan, J. L. Riggs, C. T. Schiller, E. C. Tedford, K. M. Webb, B. Windsor, J. Xia, H. L. Ypema

Joint Committee of Women in Plant Pathology \& Cultural Diversity: Chair: J. E. Fajardo. Immediate Past Chair: R. Hernandez-Martinez. Vice Chair: S. B. Ware. C. W. Bacon, M. A. Boudreau, C. T. Bull, R. Bulluck, M. L. Cromwell, A. Den Breeyen, A. Espinosa, D. G. Fernando, N.Cristina Flor, J. A. Gigot, W. Haggag, L. E. Hanson, L. Harrison, M. L. Heppler, D. M. Hinton, N. Hyder, P. Lu, R. A. Marvelli, A. E. McClean, A. L. Mila, R. O. Olatinwo, M. Peiman, M. G. Redinbaugh, M. Roca, F. Runa, E. Seyran, N. Sharma, J. L. Stephens, P. Sudarshana, L. R. Triplett, S. J. Vasquez, C. M. Vera Cruz, A. F. Watson, S. N. Wegulo, A. E. Whitfield, B. Yang

Placement: Immediate Past Chair: S. J. Vasquez. S. O. Aigbe, A. DeMarsay

Regulatory Plant Pathology: Chair: T. D. Riley. Immediate Past Chair: N. K. Osterbauer. Vice Chair: S. Rizvi. M. A. Abdelshife, T. W. Allen, C. L. Blomquist, T. N. Boratynski, V. A. Brewster, R. Bulluck, K. F. Cardwell, T. R. Gottwald, C. F. Harden, K. L. Kosta, O. Koyaolu-Salami, P. A. Nolan, C. M. Ocamb, G. L. Peterson, E. V. Podleckis, B. L. RandallSchadel, C. M. Sandlin, T. S. Schubert, C. P. Schulze, M. G. Tiffany, A. T. Tschanz, D. M. Woods, H. Xu

Teaching: Chair: H. Shew. Immediate Past Chair: D. M. Eastburn. Vice Chair: M. H. Shintaku. E. J. Braun, K. D. Cox, P. H. Flynn, C. M. Kenerley, M. A. Langham, C. Lapaire Harmon, S. C. Nelson, T. L. Niblack, M. B. Riley, M. H. Shintaku, B. S. Sipes, M. Skaria, C. M. Stiles

\section{Subject Matter Committees}

Bacteriology: Chair: S. B. Von Bodman. Immediate Past Chair: A. O. Charkowski. Vice Chair: J. D. Barak. J. R. Alfano, A. M. Alvarez, C. T. Bull, J. L. Calcote, J. Chen, J. M. Clifford, B. K. Duffy, Z. Flores, C. F. Gonzalez, J. H. Graham, R. Hernandez-Martinez, K. Jakob, J. A. Kers, S. Kim, R. Loria, F. J. Louws, S. Lu, G. Luo, M. Marutani, M. L. Miller, A. R. Records, R. M. Reedy, M. A. Savka, C. D. Smart, N. Wang, F. F. White, Y. Zhao, Y. Zhao

Biochemistry, Physiology \& Molecular Biology: Chair: R. P. Wise. Immediate Past Chair: T. K. Mitchell. Vice Chair: J. E. Flaherty. G. C. Bernard, L. J. Chapin, Y. E. Choi, Y. Dai, N. M. Donofrio, A. M. Fakhoury, A. E. Glenn, M. Gowda, D. A. Halterman, S. L. Hartney, S. Herrero, Y. Jia, M. V. Kolomiets, D. Kumar, S. Mansouri, B. B. McSpadden Gardener, P. Moffett, S. Restrepo, U. S. Sagaram, S. A. Sukno, M. R. Thon, S. R. Uppalapati, L. J. Vaillancourt, J. D. Walton, G. Wang

Biological Control: Chair: H. K. Abbas. Immediate Past Chair: S. Kiewnick. Vice Chair: S. S. Gnanamanickam. P. A. Backman, J. Borneman, W. L. Bruckart, C. T. Bull, S. Chen, A. M. Demers, B. J. Jacobsen, D. A. Kluepfel, M. S. Krause, D. Lakshman, R. P. Larkin, J. E. Loper, M. Lorito, B. B. McSpadden Gardener, R. L. Melnick, M. T. Mmbaga, F. A. Mrema, T. C. Paulitz, J. M. Raaijmakers, M. B. Rayamajhi, D. C. Sands, D. A. Schisler, S. F. Shamoun, J. W. Travis, P. Warrior, W. Wechter, G. Y. Yuen

Biotechnology: Chair: W. Wechter. Immediate Past Chair: K. Ling. Vice Chair: D. A. Kluepfel. B. Guo, D. A. Halterman, A. S. Hyten, F. Jan, M. J. Jones, Z. Kanaan-Atallah, R. R. Martin, P. Moffett, M. Peiman, A. Pepper, S. R. Scofield, Y. Wamishe, N. A. Weinsetel, B. Yang, Y. Yang

Chemical Control: Chair: D. C. Thompson. Vice Chair: W. D. Gubler. R. S. Bounds, W. Chen, A. Cochran, D. R. Cooley, J. P. Damicone, L. Fought, C. L. Kohls, H. Morton, L. A. Payan, K. M. Tubajika, B. Windsor

Crop Loss Assessment and Risk Evaluation (CARE): Chair: S. J. Pethybridge. Immediate Past Chair: D. G. Schmale. Vice Chair: D. H. Gent. A. K. Chatterjee, P. D. Esker, C. A. Hollier, S. J. Jones, M. M. Kennelly, S. J. Lambert, D. L. Long, J. S. Miller, O. Modesto Olanya, H. K. Ngugi, F. W. Nutter, P. V. Oudemans, P. A. Paul, W. F. Pfender, K. C. Steddom, J. M. Stein, K. M. Tubajika, W. W. Turechek, C. Yandoc 
Diagnostics: Chair: C. Lapaire Harmon. Immediate Past Chair: J. L. O’Mara. Vice Chair: A. Phibbs. Y. Abou Jawdah, F. M. Assis Filho, C. J. Balbalian, J. W. Beale, R. G. Bhat, W. O. Bliss, T. D. Blunt, L. G. Brown, M. E. L. Burrows, J. L. Chaky, T. C. Creswell, A. Fabritius, R. C. Hamelin, A. L. Hazelrigg, E. W. Honeycutt, M. M. Kennelly, L. Levy, S. K. Marquardt, S. R. May, R. T. McMillan, C. Nischwitz, F. M. Ochoa-Corona, J. L. O'Mara, A. J. Palmateer, M. E. Palm-Hernandez, K. L. Perry, M. Putnam, G. E. Ruhl, P. J. Shiel, C. A. Smith, K. L. Snover-Clift, F. Sun, C. L. Sutula, C. A. Webb, M. R. Williamson, S. Wright, H. Xu, K. A. Zeller, N. K. Zidack, A. D. Ziems

Diseases of Ornamental Plants: Chair: K. C. Steddom. Immediate Past Chair: C. L. Palmer. Vice Chair: D. J. Norman. F. M. Assis Filho, W. E. Copes, M. L. Daughtrey, J. L. Hall, J. R. Hartman, A. Henn, N. I. Khan, D. Lakshman, D. J. Lewandowski, M. T. Mmbaga, K. A. Plumley, B. Rao, M. G. Tiffany, A. T. Tschanz, L. E. Yakabe

Environmental Quality \& Plant Health: Immediate Past Chair: C. R. Krause. M. A. Bhatti

Epidemiology: Chair: P. A. Paul. Immediate Past Chair: P. D. Esker. Vice Chair: S. S. Savary. O. Carisse, M. I. Chilvers, E. D. De Wolf, N. S. Dufault, D. H. Gent, N. Grunwald, I. J. Holb, A. L. Mila, J. E. Molineros, U. N. Nanayakkara, H. K. Ngugi, F. W. Nutter, H. Scherm, A. C. Schuerger, A. H. Sparks, K. M. Tubajika, L. Willocquet

Forest Pathology: Chair: J. Juzwik. Immediate Past Chair: M. Kim. Vice Chair: N. B. Klopfenstein. R. S. Bounds, P. D. Brune, G. A. Chastagner, M. Chen, B. A. Edmunds, S. J. Frankel, E. M. Hansen, L. M. Haugen, N. M. Kleczewski, G. Laflamme, W. L. MacDonald, R. E. Marra, M. T. Mmbaga, C. Mohammed, J. G. O'Brien, W. D. Ostrofsky, B. A. Richardson, D. M. Rizzo, S. F. Shamoun, W. C. Shortle, P. O. Spaine, P. J. Zambino, J. W. Zanzot

Genetics: Chair: T. L. Friesen. Immediate Past Chair: K. A. Zeller. Vice Chair: P. Veronese. T. B. Adhikari, R. G. Bhat, S. L. Giammaria, V. Gnana Asir, B. Guo, R. Hernandez-Martinez, H. S. Judelson, M. R. Kerns, Z. Liu, M. A. Pastor-Corrales, H. Xue, S. Zhong

Host Resistance: Chair: G. E. Vallad. Immediate Past Chair: B. Guo. Vice Chair: Z. Chen. T. B. Adhikari, P. J. Balint-Kurti, H. Bouzar, M. C. Carrillo, T. E. Coram, F. Correa, W. Dong, J. S. Engle, S. L. Giammaria, R. C. Grube, D. A. Halterman, J. Hoogland, R. M. Hunger, S. Kang, C. S. Kousik, F. N. Lee, A. Mengistu, S. Mideros Mora, M. R. Miles, L. E. Osborne, M. A. Pastor-Corrales, J. J. Polashock, J. B. Rasmussen, M. G. Redinbaugh, J. M. Stein, J. A. Thies, P. Uribe, P. Veronese, H. Xue

Integrated Plant Disease Management: Chair: W. McFadden-Smith. Vice Chair: S. Sanogo. Y. Abou Jawdah, L. P. Berkett, G. W. Bird, P. M. Brannen, R. Bulluck, K. L. Everts, R. P. Larkin, J. J. Marois, H. Mellinger, T. M. Momol, K. L. Pernezny, P. D. Roberts, A. C. Schilder, S. M. Schneider

Mycology: Chair: C. M. Stiles. Immediate Past Chair: T. E. Chase. Vice Chair: M. E. Palm-Hernandez. G. Avila, L. E. Cadle-Davidson, W. Chen, J. C. Dianese, D. M. Eastburn, D. L. Funnell, A. J. Gevens, D. A. Glawe, S. B. Goodwin, S. A. Jordan, M. J. Kenney, D. Lakshman, C. Lapaire Harmon, S. Li, M. L. McPherson, S. Moricca, J. L. O’Mara, L. E. Osborne, A. Y. Rossman, T. L. Tarnowski, P. J. Zambino, K. A. Zeller

Mycotoxicology: Chair: D. L. Funnell. Immediate Past Chair: J. Palumbo. Vice Chair: A. M. Fakhoury. H. K. Abbas, C. W. Bacon, R. L. Brown, L. Chambliss-Bush, Z. Chen, P. J. Cotty, A. E. Desjardins, R. Dill-Macky, A. Dolezal, A. E. Glenn, B. Guo, S. Herrero, D. M. Hinton, T. A. Jackson, R. Jaime-Garcia, M. V. Kolomiets, G. Kuldau, S. Li, M. A. Mansfield, S. Mansouri, M. McDonald, G. P. Munkvold, E. R. Palencia, G. A. Payne, J. F. Plasencia, C. Probst, R. H. Proctor, A. Schaafsma, D. G. Schmale, W. Shim, C. P. Woloshuk, I. E. Yates, N. C. Zitomer
Nematology: Chair: T. A. Jackson. Immediate Past Chair: B. S. Sipes. Vice Chair: S. L. Meyer. R. Bulluck, S. Chen, E. L. Davis, T. A. Forge, C. S. Johnson, N. Kokalis-Burelle, R. Levin, M. G. Mitchum, T. L. Niblack, F. Sun, G. L. Tylka, K. Wang, J. K. Welch, A. Westphal, L. Xing, A. D. Ziems

Pathogen Resistance: Chair: N. Peres. Immediate Past Chair: W. F. Wilcox. J. E. Adaskaveg, J. P. Damicone, H. C. Forster, W. D. Gubler, W. D. Koeller, D. B. Langston, P. I. Lewis, M. T. McGrath, H. Morton, G. Olaya, L. W. Timmer, W. L. Wiebe, F. P. Wong, C. A. Wyenandt, C. Xiao

Phyllosphere Microbiology: Chair: M. T. Brandl. Vice Chair: V. Toussaint. B. Balogh, G. A. Beattie, R. G. Bhat, K. R. Everett, B. C. Freeman, J. L. Jacobs, K. Jakob, K. B. Johnson, S. E. Lindow, W. F. Mahaffee, P. S. McManus, R. T. McMillan, B. B. McSpadden Gardener, V. Philion, P. Pusey, M. Saunders, V. O. Stockwell, G. W. Sundin

Plant Pathogen and Disease Detection: Chair: I. E. Tzanetakis. Immediate Past Chair: M. G. Tiffany. Vice Chair: R. G. Bhat. A. Ali, T. D. Blunt, A. Fabritius, J. M. French, J. P. Gaydos, S. L. Giammaria, G. P. Hoyos, C. J. Kahlke, M. Kenganal, L. Levy, H. R. Pappu, K. L. Perry, P. J. Shiel, C. D. Smart

Postharvest Pathology: Chair: E. C. Tedford. Immediate Past Chair: J. E. Adaskaveg. Vice Chair: G. J. Holmes. J. A. Bartz, R. S. Bounds, P. D. Brune, B. D. Bruton, A. Cochran, W. S. Conway, H. Dou, B. A. Edmunds, H. C. Forster, W. J. Janisiewicz, L. Kanetis, Y. Kim, Y. Luo, J. Mercier, T. J. Michailides, D. B. Prusky, P. L. Sholberg, J. L. Smilanick, D. Sugar, K. M. Tubajika, C. Xiao

Seed Pathology: Chair: L. J. du Toit. Immediate Past Chair: W. L. Wiebe. Vice Chair: S. A. Heuchelin. A. M. Alvarez, K. S. Arthur, C. C. Block, H. Bouzar, P. R. Brown, L. D. Butler, L. Carter, A. C. Castro, M. A. Draper, A. Fabritius, E. W. Gatch, R. L. Gilbertson, S. A. Heuchelin, P. T. Himmel, G. P. Hoyos, K. L. Johnson, W. Kaneshiro, H. Koenraadt, C. J. Kurowski, G. L. Lamka, Z. Lan, D. C. McGee, M. E. Meadows, G. P. Munkvold, G. Olaya, L. M. Shepherd, A. B. Thornton, M. G. Tiffany, R. R. Walcott, S. K. Walker, K. M. Webb

Soil Microbiology and Root Diseases: Chair: B. M. Wu. Immediate Past Chair: A. T. Dyer. Vice Chair: C. A. Strausbaugh. Z. Atallah, G. T. Browne, R. Bulluck, A. O. Charkowski, D. A. Doll, K. L. Everts, E. J. Fichtner, X. Gao, N. Grunwald, B. K. Gugino, M. Jimenez-Gasco, D. Lakshman, F. J. Louws, O. V. Mavrodi, N. Ochiai, T. C. Paulitz, H. Ramasubramaniam, B. S. Richter, L. S. Schmidt, S. R. Uppalapati, G. E. Vallad, D. M. Weller, A. Westphal, L. Xing

Tropical Plant Pathology: Chair: J. T. Tambong. Immediate Past Chair: M. P. Grisham. Vice Chair: K. A. Garrett. S. O. Aigbe, C. A. Angel, W. O. Bliss, R. H. Brlansky, F. E. Brooks, J. L. Calcote, L. A. Calvert, J. Cobb, F. Correa, S. L. Giammaria, C. E. Jahn, M. Montero Astua, F. M. OchoaCorona, B. L. Randall-Schadel, B. S. Sipes, R. A. Valverde

Turfgrass Pathology: Chair: J. E. Kaminski. Vice Chair: H. C. Wetzel. M. L. Agnew, T. D. Blunt, M. J. Boehm, D. Y. Han, B. J. Horvath, K. S. Jordan, G. Jung, M. M. Kennelly, J. P. Kerns, U. N. Nanayakkara, S. R. Parker, D. H. Perry, B. Rao, D. L. Smith, M. Tomaso-Peterson, L. P. Tredway, J. R. Young

Virology: Chair: D. J. Lewandowski. Immediate Past Chair: R. W. Hammond. Vice Chair: R. L. Jordan. J. A. Abad, R. Acosta-Leal, A. Ali, C. A. Angel, F. M. Assis Filho, M. E. L. Burrows, A. B. Cole, J. Hammond, A. M. Idris, R. L. Jordan, M. A. Langham, R. T. Lartey, K. Ling, C. J. Maroon-Lango, U. K. Melcher, B. Meng, L. G. Nemchinov, H. R. Pappu, N. A. Rayapati, N. L. Robertson, G. Roy, W. Schneider, I. E. Tzanetakis, M. T. Watson, R. A. Welliver, A. E. Whitfield 